\title{
High-frequency characterization of an optical phase modulator with phase modulation-to-intensity modulation conversion in dispersive fibers
}

\author{
ZHANG ShangJian", LU RongGuo, CHEN DeJun, LIU Shuang \& LIU Yong \\ State Key Laboratory of Advanced Optical Communication Systems and Networks, School of Optoelectronic Information, University of Electronic \\ Science \& Technology of China, Chengdu 610054, China
}

Received December 12, 2011; accepted March 8, 2012

\begin{abstract}
We propose a new method for characterizing optical phase modulators based on phase modulation-to-intensity modulation (PM-to-IM) conversion in dispersive fibers. The fiber dispersion spectrally alters the relative phasing of the phase-modulated signal and leads to the PM-to-IM conversion, which is extended to measure the modulation efficiency of optical phase modulators. In the demonstration, the frequency-dependent modulation index and half-wave voltage are experimentally measured for a commercial phase modulator. Compared with conventional methods, the proposed method works without the restriction of small-signal operations, and allows swept-frequency measurement with high resolution and accuracy by using a vector network analyzer.
\end{abstract}

electro-optic modulators, microwave photonics, optical fiber dispersion, phase modulation

Citation: Zhang S J, Lu R G, Chen D J, et al. High-frequency characterization of an optical phase modulator with phase modulation-to-intensity modulation conversion in dispersive fibers. Chin Sci Bull, 2012, 57: 2929-2933, doi: 10.1007/s11434-012-5210-3

Optical phase modulation can eliminate bias drifting and generate inherent out-of-phase sidebands compared with Mach-Zehnder intensity modulation, which has attracted significant interest in microwave photonic signal processing [1], such as photonic microwave filtering [2,3] and photonic microwave frequency measurement $[4,5]$. In phase-modulation-based microwave photonics systems, the modulation efficiency of the optical phase modulator, the modulation index and the half-wave voltage influence the overall highfrequency performance. This is especially true at broadband microwave operation, which should be critically characterized to evaluate and optimize the system. However, optical phase modulators only change the optical phase, which makes it difficult to measure directly the modulation efficiency with developed intensity-sensitive instruments [6].

Many measurement techniques have been proposed such as optical spectrum analysis [6], optical frequency discrimination [7], self-heterodyne method [8], and Sagnac loop

*Corresponding author (email: sjzhang@uestc.edu.cn) method [9]. Optical spectrum analysis has a very simple configuration, which is to measure the optical spectrum of the phase-modulated signal. Nevertheless, it is a point-topoint measurement, and its resolution is limited by the optical spectrum analyzer (about $0.01 \mathrm{~nm}$ ) [9]. Optical frequency discrimination uses a Fabry-Perot (FP) interferometer to make a frequency-to-amplitude conversion. However, it suffers from the nonlinear slope of the optical frequency discriminator. The Sagnac loop method is based on the delayed interference of bidirectional modulations and achieves swept-frequency measurement with high resolution, and is applicable to small-signal operation and requires an envelope extraction.

As is known, phase-modulated signals can be converted to intensity modulation by inserting a dispersive device after phase modulation $[1-5,10]$. Phase modulation-to-intensity modulation (PM-to-IM) conversion has been widely used to demodulate the phase-modulated optical signal in the receivers of microwave photonic systems [1]. In this paper, we investigate PM-to-IM conversion in the context of char- 
acterizing optical phase modulators, and propose a new method for measuring the modulation efficiency through the PM-to-IM conversion in fold-back dispersive fiber paths. We theoretically analyze the evolution of phase-modulated signals in the dispersive fiber and present the measurement principle with a fold-back configuration to demonstrate our method. The frequency-dependent modulation index and half-wave voltage are experimentally measured for a commercial phase modulator. Our method works without the restriction of small-signal operation, and allows sweptfrequency measurement with high resolution and accuracy by using a network analyzer, which avoids using an unstable interferometer or additional envelope extraction.

\section{Operating principle}

As is shown in Figure 1(a), an optical carrier at an angular frequency $\omega_{0}$ is phase-modulated by an electrical sinusoidal signal at an angular frequency $\omega_{e}$. The phase-modulated optical signal can be expanded in terms of the Bessel functions

$$
\mathrm{e}_{1}(t)=\mathrm{e}^{j \omega_{0} t+j m \cos \left(\omega_{e} t\right)}=\sum_{n=-\infty}^{+\infty} j^{n} J_{n}(m) \mathrm{e}^{j \omega_{0} t+j n \omega_{e} t},
$$

where $J_{n}(\bullet)$ is the $n$ th-order Bessel function of the first kind and $m$ is the modulation index of the phase modulator. It is obvious that phase modulation generates multiple optical sidebands at both sides of the optical carrier with frequency spaced by $\omega_{e}$ and phase shifted by $\pi / 2$.

After dispersion propagation, an additional phase shift is introduced to each optical sideband depending on its frequency offset relative to the optical carrier. The phase shift induced by the fiber dispersion with length $L$ can be approximately represented by

$$
\phi\left(\omega_{0}+n \omega_{e}\right)=\left(\beta_{0}+\beta_{1} n \omega_{e}+\beta_{2} n^{2} \omega_{e}^{2} / 2\right) L .
$$

In the expansion, the first two terms give rise to a fixed phase shift and propagation delay, and the third term denotes the group velocity dispersion, in which the dispersion coefficient $\beta_{2}$ relates to the fiber dispersion parameter $D$ by $D=-2 \pi \beta_{2} c \omega_{0}^{2}$. Assuming fiber loss contributes only with a constant factor $\alpha$, the optical signal after dispersion propagation can be written as

$$
\mathrm{e}_{2}(t)=\sum_{n=-\infty}^{+\infty} j^{n} J_{n}(m) \mathrm{e}^{\alpha / 2} \mathrm{e}^{j \phi\left(\omega_{0}+n \omega_{e}\right)} \mathrm{e}^{j \omega_{0} t+j n \omega_{e} t}
$$

and the optical intensity after dispersion propagation is given by

$$
\begin{aligned}
i(t) & =\mathrm{e}_{2}(t) \cdot \mathrm{e}_{2}^{*}(t) \\
& =\mathrm{e}^{\alpha} \sum_{k=-\infty}^{+\infty} j^{k} \mathrm{e}^{j k \omega_{e} t+j \varphi_{1}+j k \varphi_{2}} \sum_{n=-\infty}^{+\infty} J_{n+k}(m) J_{n}(m) \mathrm{e}^{j 2 n \varphi_{2}},
\end{aligned}
$$

with $\varphi_{1}=\beta_{1} L k \omega_{e}$ and $\varphi_{2}=\beta_{2} L k \omega_{e}{ }^{2} / 2$. Applying Graf's additional formula of Bessel functions [10,11], the expression for the received optical intensity can be further reduced to

$$
i(t)=\mathrm{e}^{\alpha}\left[1+2 \sum_{k=1}^{+\infty}(-1)^{k} J_{k}\left(2 m \sin \varphi_{2}\right) \cos \left(k \omega_{e} t+\varphi_{1}\right)\right] \text {. }
$$

It can be seen from eq. (5) that the fiber-dispersion-induced phase shift alters the phase spectrum of the phase-modulated signal, and leads to PM-to-IM conversion. To develop a practical method, we focus on the fundamental component in eq. (5). We define the ratio of the first-order harmonic component to the dc component as the strength of the firstorder harmonic (SFH) given by

$$
I_{1}=2\left|J_{1}\left(2 m \sin \left(\beta_{2} L \omega_{e}^{2} / 2\right)\right)\right|,
$$

which shows that the SFH function periodically achieves null at the notch frequencies corresponding to

$$
\omega_{n u}^{2}=2 u \pi /\left|\beta_{2} L\right|,(u=0,1,2 \cdots) .
$$

The notch frequency only depends on the fiber dispersion, and so it can be used to indicate the fiber dispersion. Once

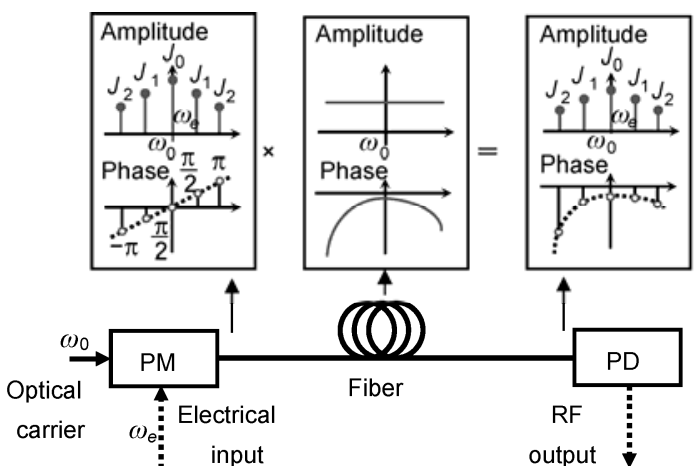

(a)

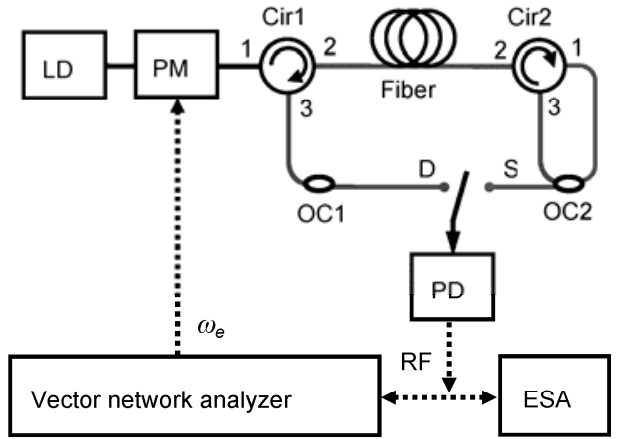

(b)

Figure 1 (a) Scheme of dispersion-induced PM-to-IM conversion and (b) diagram of the proposed fold-back configuration for measuring the modulation efficiency of phase modulators. PM, phase modulator; ESA, electrical spectrum analyzer; Cir, circulator; OC, optical coupler; PD, photodiode. 
the fiber dispersion is determined from the characteristic notch frequency, the modulation index can be determined from the measured SFH, and its high-frequency degradation can be obtained by the swept-frequency measurement with a vector network analyzer owing to the fundamental component measurement [12].

For the measuring accuracy, we investigate the error dependence of the modulation index on the dispersion uncertainty by the error transfer factor

$$
F=\left|\frac{\Delta m}{m} / \frac{\Delta L \beta_{2}}{L \beta_{2}}\right|=\left|\frac{\beta_{2} L \omega_{e}^{2}}{2} \operatorname{ctg}\left(\frac{\beta_{2} L \omega_{e}^{2}}{2}\right)\right|,
$$

which is deduced from the total differential of the SFH function. For $F<1$, the uncertainty of the modulation index is less than that of the fiber dispersion, whereas for $F>1$, the uncertainty is larger and gives rise to a large error of the modulation index. Therefore, the fiber dispersion should be carefully chosen to feature $F \leqslant 1$ as $\left|\beta_{2} L \omega_{x}{ }^{2}\right| \leqslant 4.0575$ with a maximum angular frequency $\omega_{x}$. That is to say, all the non-zero-frequency notches should be out of the measuring frequency range, which does not agree with the requirement of determining the fiber dispersion according to eq. (7).

To deal with the problem, we introduce a fold-back configuration in Figure 1(b), where the round-trip dispersion is double the single-trip dispersion. The fiber length is set as

$$
\pi \leqslant\left|\beta_{2} L \omega_{x}^{2}\right| \leqslant 4.0575
$$

to allow an optimum single-trip dispersion for measuring the modulation index. Meanwhile, the fiber length also ensures enough round-trip dispersion for accurately determining the fiber dispersion from the non-zero-frequency notch within the measuring frequency range.

\section{Experiment and results}

To demonstrate our scheme, we experimentally characterize a commercial phase modulator using the setup shown in
Figure 1(b). According to eq. (9), the optimum fiber dispersion should be $199 \mathrm{ps}^{2} \leqslant\left|\beta_{2} L\right| \leqslant 257 \mathrm{ps}^{2}$ in the frequency range of $20 \mathrm{GHz}$, which corresponds to a $9.2-$ to $11.8-\mathrm{km}$ standard single-mode fiber operated at $1550 \mathrm{~nm}$. In our experiment, the optical carrier from a DFB laser diode at $1550.2 \mathrm{~nm}$ is first sent to a $\mathrm{LiNbO}_{3}$ phase modulator. The phase-modulated optical signal is then coupled into an 11.5-km single-mode fiber via an optical circulator (Cir1). At the end of the fiber, another optical circulator (Cir2) is terminated to loop back the optical signal. In the case of single-trip dispersion, the measured signal comes from port 1 of Cir2 via an optical coupler (OC2). In the case of round-trip dispersion, we measure the optical signal at port 3 of Cir1. The optical coupler (OC1) is used to compensate for the fold-back path to keep the relationship between the single-trip and round-trip dispersions.

The optical intensity after fiber propagation is detected by a New Focus $45-\mathrm{GHz}$ photodiode, which has a flat responsivity within a $20-\mathrm{GHz}$ frequency range and its error factor is negligible. With a full two-port calibration, an HP8720D vector network analyzer is used to generate a swept-frequency signal and measure the first-order harmonic component of the optical intensity. As the dc component is the same for all swept frequencies, we measure it with an R\&S FSU43 electrical spectrum analyzer at one frequency e.g., at $8 \mathrm{GHz}$, as reference, from which the measured SFH is obtained.

As is shown in Figure 2(a), the measured SFHs under the single-trip dispersion achieve only a dc notch, while the results under the double-trip dispersion achieve a non-zerofrequency notch at $17.87 \mathrm{GHz}$ besides a dc notch, which indicates a round-trip dispersion of $498.4 \mathrm{ps}^{2}$ and a single-trip dispersion of $249.2 \mathrm{ps}^{2}$. The error transfer factor for both cases is calculated and plotted in Figure 2(b). One can see that the error transfer factor reaches maximum at 17.87 $\mathrm{GHz}$ (notch frequency) in the round-trip case, whereas it keeps no more than 1 within the whole frequency range in the single-trip case. It is worth noting that the error transfer factor approximately equals 1 around zero frequency;
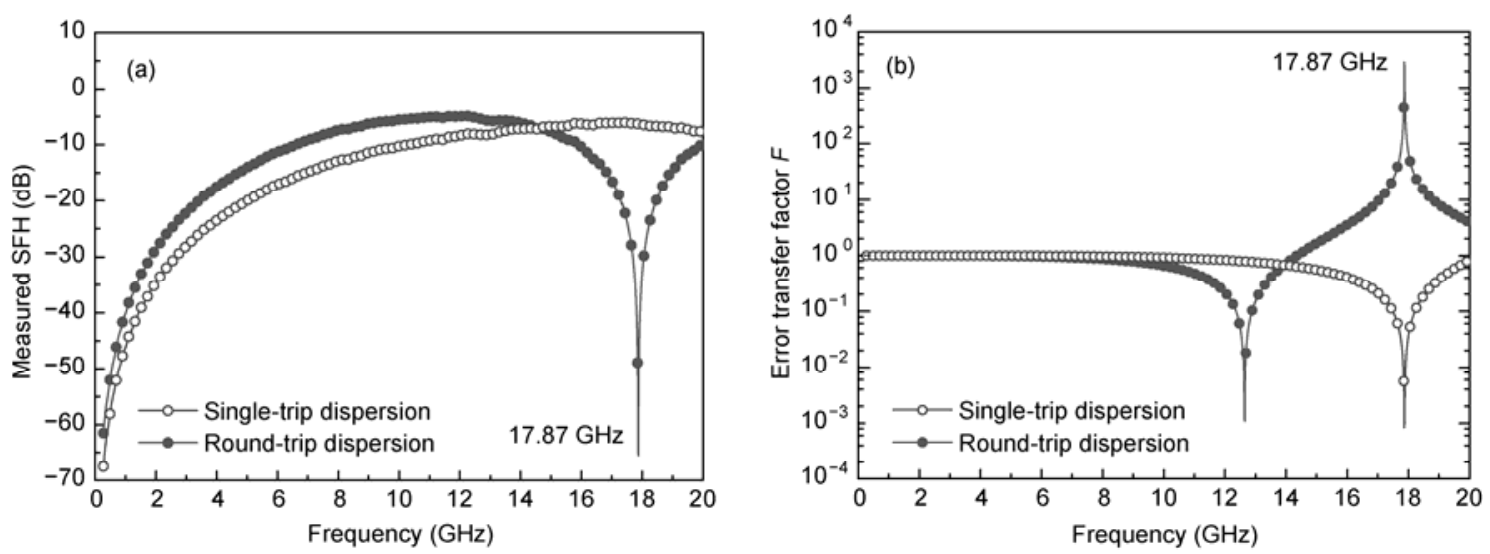

Figure 2 (a) Measured SFH and (b) error transfer factors for different cases of dispersion, where a $11.5 \mathrm{~km}$ standard single-mode fiber is used. 
that is to say, the dc notch has little influence on the measuring accuracy of the modulation index. The error factor reaches minimum at $12.63 \mathrm{GHz}$ for the round-trip case and $17.87 \mathrm{GHz}$ for the single-trip case, which indicates the minimum error of the modulation index at these frequencies.

The frequency-dependent modulation index is determined from the measured SFH based on eq. (6), and results for the single-trip and round-trip dispersions are shown in Figure 3 for comparison. We see that the errors around the notch frequency in the round-trip case are largely suppressed, and the single-trip case shows obvious improvement without any unreasonable error. The modulation index of the phase modulator decreases from 0.55 to $0.21 \mathrm{rad}$ when the modulation frequency increases from 0.05 to 20 GHz. The phase modulator is also measured using the conventional optical spectrum analysis for comparison, as shown in Figure 3, where the frequency space of about 1 $\mathrm{GHz}$ and lower frequency of about $4 \mathrm{GHz}$ are limited by the resolution of the OSA and the linewidth of the laser source, respectively. Nevertheless, our results are in good agreement with those obtained using the conventional method.

Furthermore, the half-voltage of the phase modulator is characterized with the same setup. The electrical reflection coefficient $\Gamma_{L}$ of the phase modulator is simultaneously measured with the calibrated vector network analyzer, and the input impedance $Z_{L}$ of the modulator and its electrical driving amplitude $V_{e}$ are obtained using $Z_{L}=50\left(1+\Gamma_{L}\right) /$ $\left(1-\Gamma_{L}\right)$ and $V_{e}^{2}=2\left|Z_{L}\right| P_{e}$, respectively. Therefore, the halfwave voltage of the modulator can be determined with the help of $V_{\pi}=\pi V_{e} / m$, which increases from 3.2 to $7.7 \mathrm{~V}$ with a modulation frequency up to $20 \mathrm{GHz}$.

\section{Conclusion}

During the experiment, our measurements had stable and

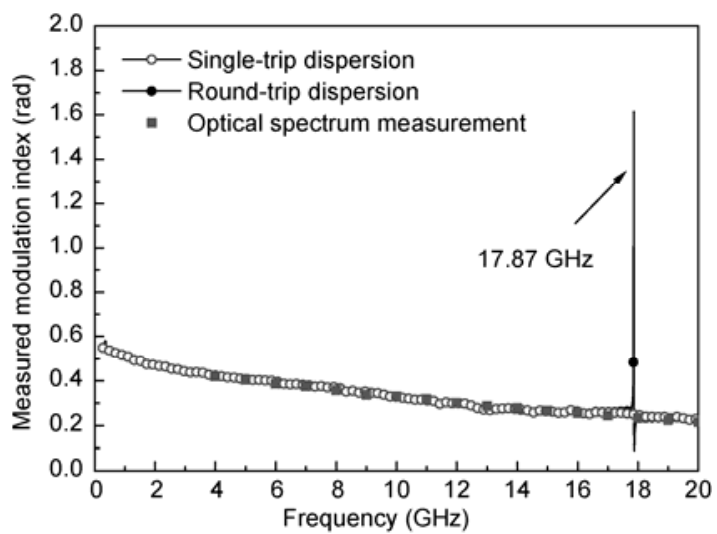

Figure 3 Measured modulation index under single-trip dispersion (open circles) and round-trip dispersion (filled circles), where filled squares represent the results obtained using optical spectrum analysis. repeatable results because the optical carrier and its sidebands are affected by the same fiber impairments. We did our measurement at an electrical power of $7 \mathrm{dBm}$, which is applicable to other driving levels since our method does not assume any small-signal operation [10,11]. Moreover, the method can be extended to other wavelengths and other dispersive fibers as long as the operation meets the dispersion requirement.

The frequency-dependent modulation index and halfvoltage confirm the aforementioned degradation of modulation efficiency at high-frequency modulation. It has been noted that the degradation is mainly due to (1) the phasevelocity mismatches between the optical wave and the microwave and (2) the impedance mismatches between the microwave source and the modulator $[13,14]$. Our measurement obviously provides much detailed information for characterizing and optimizing both phase modulators and phase-modulation-based systems.

In summary, we proposed and demonstrated a simple and effective method to characterize phase modulators based on PM-to-IM conversion in fold-back dispersive paths. The frequency-dependent modulation index and half-wave voltage can be accurately obtained by measuring the optical intensity of the phase-modulated signal after experiencing dispersion propagation. Our method benefits from the high resolution and swept frequency of developed electricaldomain measurement techniques, which largely enhance the characterization of electro-optic phase modulators.

This work was supported by the National Basic Research Program of China (2011CB301705, 2012CB315701), the Program for New Century Excellent Talents in University (NCET-11-0069) and the National Natural Science Foundation of China (60907008, 60925019, 61090393).

1 Yao J. Microwave photonics. J Lightw Technol, 2009, 27: 314-335

2 Zeng F, Yao J. Investigation of phase-modulator-based all-optical bandpass microwave filter. J Lightw Technol, 2005, 23: 1721-1728

3 Yu Y, Xu E, Dong J, et al. Switchable microwave photonic filter between high $\mathrm{Q}$ bandpass filter and notch filter with flat passband based on phase modulation. Opt Express, 2010, 18: 25271-25282

4 Zhou J, Fu S, Shum P, et al. Photonic measurement of microwave frequency based on phase modulation. Opt Express, 2009, 17: 72177221

5 Zhou X, Pan S, Yao J. Instantaneous microwave frequency measurement with improved measurement range and resolution based on simultaneous phase modulation and intensity modulation. J Lightw Technol, 2009, 27: 5314-5320

6 Shi Y, Yan L, Willner A E. High-speed electrooptic modulator characterization using optical spectrum analysis. J Lightw Technol, 2003, 21: $2358-2367$

7 Levesque M, Tetu M, Tremblay P, et al. A novel technique to measure the dynamic response of an optical phase modulator. IEEE Trans Instrum Meas, 1995, 44: 952-957

8 Campbell J A, Knoesen A, Yankelevich D R. Measurement of the modulation efficiency of an optical phase modulator using a selfhomodyne receiver. IEEE Photon Technol Lett, 2002, 14: 1330-1332

9 Chan E H W, Minasian R A. A new optical phase modulator dynamic response measurement technique. J Lightw Technol, 2008, 26: 2882- 
2888

10 Chi H, Zou X, Yao J. Analytical models for phase-modulation- based microwave photonic systems with phase modulation to intensity modulation conversion using a dispersive device. J Lightw Technol, 2009, 27: 511-521

11 Walker N G, Walker D, Smith I C. Efficient millimeter-wave signal generation through FM-IM conversion in dispersive optical fiber links. Electron Lett, 1992, 28: 2027-2028
12 Liu Y, Chen S F, Wang X, et al. Overall optimization of highspeed semiconductor laser modules. Chin Sci Bull, 2009, 54: 36973703

13 Liao Y, Zhou H, Meng Z. Modulation efficiency of a linbo3 waveguide electro-optic intensity modulator operating at high microwave frequency. Opt Lett, 2009, 34: 1822-1824

14 Lu R G, Liao J K, Tang X G, et al. Optimization design for polymeric S-shaped ridge waveguide. Chin Sci Bull, 2010, 55: 1834-1839

Open Access This article is distributed under the terms of the Creative Commons Attribution License which permits any use, distribution, and reproduction in any medium, provided the original author(s) and source are credited. 